\title{
Polynomials and General Degree-Based Topological Indices of Generalized Sierpinski Networks
}

\author{
Chengmei Fan, ${ }^{1}$ M. Mobeen Munir ${ }^{1},{ }^{2}$ Zafar Hussain, ${ }^{3}$ Muhammad Athar, ${ }^{4}$ \\ and Jia-Bao Liu $\mathbb{i D}^{5}$ \\ ${ }^{1}$ College of Modern Service Industry, Hefei College of Finance and Economics, Hefei 230601, China \\ ${ }^{2}$ Department of Mathematics, University of the Punjab, Lahore, Pakistan \\ ${ }^{3}$ Department of Mathematics and Statistics, the University of Lahore, Lahore, Pakistan \\ ${ }^{4}$ Department of Mathematics, Division of Science and Technology, University of Education Lahore, Lahore, Pakistan \\ ${ }^{5}$ School of Mathematics and Physics, Anhui Jianzhu University, Hefei 230601, China \\ Correspondence should be addressed to M. Mobeen Munir; mmunir@ue.edu.pk
}

Received 16 November 2020; Revised 8 January 2021; Accepted 16 January 2021; Published 9 February 2021

Academic Editor: M. Irfan Uddin

Copyright (c) 2021 Chengmei Fan et al. This is an open access article distributed under the Creative Commons Attribution License, which permits unrestricted use, distribution, and reproduction in any medium, provided the original work is properly cited.

\begin{abstract}
Sierpinski networks are networks of fractal nature having several applications in computer science, music, chemistry, and mathematics. These networks are commonly used in chaos, fractals, recursive sequences, and complex systems. In this article, we compute various connectivity polynomials such as $M$-polynomial, Zagreb polynomials, and forgotten polynomial of generalized Sierpinski networks $S_{k}^{n}$ and recover some well-known degree-based topological indices from these. We also compute the most general Zagreb index known as $(\alpha, \beta)$-Zagreb index and several other general indices of similar nature for this network. Our results are the natural generalizations of already available results for particular classes of such type of networks.
\end{abstract}

\section{Introduction}

Networks are studied in graph theory as spacial graphs having nice properties. A fractal is a geometric structure showing capabilities of self-similarity and repetition throughout the structure. Some commonly used fractalnatured networks appear useful in the study of complex systems present in both natural and artificial systems such as computer systems, music, brain, and social networks, allowing further development of the field in network science. These networks are largely used to capture images of complex structures and predict behaviour of biological processes like nerve dendrites and growth pattern of bacteria in any culture. These networks are very close to WK-recursive networks used in the design and implementation of local area networks as well as parallel processing systems. A special case of generalized Sierpinski graphs is the duality of Apollonian network, which displays the prominent scalefree small-world characteristics as observed in various real networks [1]. Because of diverse properties, these networks have been a subject matter of recent research.

A topological invariant is a mathematical property that remains invariant under the isomorphism of topological structures. In chemical graph theory, one is in search of the most general graph invariant that can capture at most all numerical descriptors of the structure. A new polynmial graph invariant, M-polynomial, was introduced in [2] by Deutsch and Klavžar. This invariant plays similar role in determining degree-based indices as played by Hosoya polynomial in determining distancebased indices.

Applications of the aforementioned polynomial can be traced in mathematical chemistry and pharmacology. Many degree-based topological indices can be derived using successive operations of differentiation and integration. The most interesting application of the $M$-polynomial is that almost all degree-based graph invariants, which are used to predict physical, chemical, and pharmacological properties 
of organic molecules, can be recovered from it (for more information, see [3-9]).

The $M$-polynomial and related topological indices have been studied for several classes of graphs. In 2015, Deutsch and Klavžar gave $M$-polynomial, first Zagreb, and second Zagreb indices of polyomino chains, starlike trees, and triangulenes [2]. In 2017, Mobeen gave $M$-polynomial and several degree-based topological indices of titania nanotubes [10], of triangular boron nanotubes [11], and of Jahangir graph [12]. In 2017, Ajmal gave $M$-polynomial and topological indices of generalized prism network [13]. The authors computed $M$-polynomial and related indices of some benezoid systems in [14], hex-derived network in [15], and $\mathrm{V}$-phenylenic nanotubes and nanotori [16]. Today, the computation of this polynomial is considered as one of the most important areas of research in the fields of molecular computing and degree-based indices.

Several degree-based topological indices, which play important role in mathematical chemistry, can be recovered from the $M$-polynomial. The most famous degreebased index is the Randić index which was introduced by Milan Randić in 1975 [17]. It is often used in cheminformatics for investigations of organic compounds (for more information, see [18-20]). Later in 1998, working independently, Amic [21] and Bollobas-Erdos [22] proposed the generalized Randić index (for more information, see $[23,24])$. Gutman and Trinajstić introduced first Zagreb and second Zagreb indices in 1972 [25]. The augmented Zagreb index was proposed by Furtula in 2010 in [26] and is useful for computing heat of information of alkanes $[18,27]$. To know more about topological indices, their computing, and their applications, we refer the reader to $[2,8,11,12,28-33]$.

This article is organized as follows. In Section 2, we give definitions of graph, topological indices, $M$-polynomial, and Sierpinski networks. The main results about the Sierpinski networks are given in Section 3; this section also covers the results about the topological indices.

\section{Preliminary Notes}

Definition 1. A graph $G$ is a pair $(V, E)$, where $V$ is the set of vertices and $E$ is the set of edges. Any standard text on graph theory can be used as fundamental concepts related to graph and network theory.

The edge $e$ between two vertices $u$ and $v$ is denoted by $(u, v)$. The degree of a vertex $u$, denoted by $d_{u}$, is the number of edges incident to it. A path from a vertex $v$ to a vertex $w$ is a sequence of vertices and edges that starts from $v$ and stops at $w$. The number of edges in a path is the length of that path. A graph is said to be connected if there is a path between any two of its vertices.

A molecular graph is a representation of a chemical compound in terms of graph theory. Specifically, molecular graph is a graph whose vertices correspond to (carbon) atoms of the compound and whose edges correspond to chemical bonds [34]. For our computational models, we consider graphs which are hydrogen suppressed.
In the following, by $G$ we shall mean a connected graph, $E$ its edge set, $V$ its vertex set, $e=(u, v)$ its edge joining the vertices $u$ and $v$, and $d_{u}$ degree of its vertex $u$.

Definition 2 (see [2]). The M-polynomial of $G$ is $M(G ; x, y)=\sum_{i \leq j} m_{i j} x^{i} y^{j}$, where $m_{i j}$ is the number of edges $e=(u, v)$ of $G$ with $d_{u}=i$ and $d_{v}=j$.

Definition 3. The first Zagreb polynomial, second Zagreb polynomial, and forgotten polynomial of $G$ are, respectively, defined as $M_{1}(G ; x)=\sum_{u v \varepsilon E(G)} x^{\left[d_{u}+d_{v}\right]}, \quad M_{2}(G ; x)=$ $\sum_{u v \varepsilon E\left(S_{4}^{n}\right)} x^{\left[d_{u} \times d_{v}\right]}$, and $F(G ; x)=\sum_{u v \varepsilon E(G)} x^{\left[d_{u}^{2}+d_{v}^{2}\right]}$.

A function $I$ which assigns to every connected graph $G$ a unique number $I(G)$ is called a graph invariant. Instead of the function $I$, it is custom to say the number $I(G)$ as the invariant.

Definition 4 (see [34]). An invariant of a molecular graph which can be used to determine structure-property or structure-activity correlation is called the topological index. A topological index is an invariant of the graph.

A topological index is said to be degree-based if it depends on degrees of the vertices of the graph.

The following are definitions of some degree-based indices that have connection with the $M$-polynomial.

Definition 5. The first Zagreb, second Zagreb, and second modified Zagreb indices of $G$ are defined, respectively, by $M_{1}(G)=\sum_{e \in E}\left(d_{u}+d_{v}\right), \quad M_{2}(G)=\sum_{e \in E} d_{u} \times d_{v}, \quad$ and $M M_{2}(G)=\sum_{e \in E} 1 /\left(d_{u} \times d_{v}\right)$.

Definition 6. The generalized Randic and reciprocal generalized Randic indices of $G$ are defined, respectively, by $R_{\alpha}(G)=\sum_{e \in E}\left(d_{u} \times d_{v}\right)^{\alpha}$ and $R R_{\alpha}(G)=\sum_{e \in E} 1 /\left(d_{u} \times d_{v}\right)^{\alpha}$.

Definition 7. The symmetric division index of $G$ is defined by the relation

$$
\operatorname{SSD}(G)=\sum_{e \in E}\left\{\frac{\min \left(d_{u}, d_{v}\right)}{\max \left(d_{u}, d_{v}\right)}+\frac{\max \left(d_{u}, d_{v}\right)}{\min \left(d_{u}, d_{v}\right)}\right\} .
$$

Definition 8. The harmonic, inverse sum, augmented Zagreb, and forgotten indices are defined, respectively, by $H(G)=\sum_{u v \varepsilon E(G)} 2 /\left(d_{u}+d_{v}\right), \quad I(G)=\sum_{u v \varepsilon E(G)}\left(\left(d_{u} d_{v}\right) /\right.$ $\left.\left(d_{u}+d_{v}\right)\right), \quad A(G)=\sum_{u v \varepsilon E(G)}\left\{\left(d_{u} d_{v}\right) /\left(d_{u}+d_{v}-2\right)\right\}^{3}, \quad$ and $F(G)=\sum_{u v \varepsilon E\left(S_{4}^{n}\right)}\left[d_{u}^{2}+d_{v}^{2}\right]$.

A remarkable property of the $M$-polynomial is that all the above degree-based indices can be recovered from it, using the relations given in Table 1 .

Another approach to reach at many Zagreb indices is to compute $(\alpha, \beta)$-Zagreb index introduced by Azhari et al. in [35]. It can be defined as 
TABLe 1: Relations of indices with $M$-polynomial.

\begin{tabular}{lc}
\hline Index & Relation with the $M$-polynomial \\
\hline First Zagreb & $M_{1}(G)=\left(D_{x}+D_{y}\right)(M(G))_{x=y=1}$ \\
Second Zagreb & $M_{2}(G)=\left(D_{x} \times D_{y}\right)(M(G))_{x=y=1}$ \\
Modified second Zagreb & $M_{2}(G)=\left(S_{x} \times S_{y}\right)(M(G))_{x=y=1}$ \\
Generalized Randić & $R_{\alpha}(G)=\left(D_{x}^{\alpha} \times D_{y}^{\alpha}\right)(M(G))_{x=y=1}$ \\
Reciprocal generalized Randić & $R R_{\alpha}(G)=\left(S_{x}^{\alpha} \times S_{y}^{\alpha}\right)(M(G))_{x=y=1}$ \\
Symmetric division & $\operatorname{SDD}(G)=\left(D_{x} S_{y}+D_{y} S_{x}\right)(M(G))_{x=y=1}$ \\
Harmonic & $H(G)=2 S_{x} J(M(G))_{x=1}$ \\
Inverse sum & $I(G)=S_{x} J D_{x} D_{y}(M(G))_{x=1}$ \\
Augmented Zagreb & $A(G)=S_{x}^{3} Q_{-2} J D_{x}^{3} D_{y}^{3}(M(G))_{x=1}$ \\
\hline & $D_{x}=x(\partial / \partial x) M(G), D_{y}=y(\partial / \partial y) M(G)$ \\
Where & $S_{x}=\int_{0}^{x}(M(G ; t, y) / t) \mathrm{d} t, S_{y}=\int_{0}^{y}(M(G ; x, t) / t) \mathrm{d} t$ \\
& $J=M(G ; x, x), Q_{-2}=x^{-2} M(G ; x, y)$ \\
\hline
\end{tabular}

$$
Z_{\alpha, \beta}=\sum_{u v \in E(G)}\left[(d u)^{\alpha}(d v)^{\beta}+(d u)^{\beta}(d v)^{\alpha}\right] .
$$

Sarkar et al. [36] computed $Z_{\alpha, \beta}$ for some derived networks. The authors computed this index for $\mathrm{V}$-phenylenic nanotubes and nanotori molecules [37] and circumcoronene series of benzenoid in [38]. This is somehow more general index, and several other indices can be calculated evaluating it at certain values. The following table establishes relation of other Zagreb indices as particular cases of this general Zagreb index. The redefined Zagreb index was first introduced in 2013 by Ranjini et al. [39] and is defined as

$$
\operatorname{ReZM}(G)=\sum_{u v \in E(G)}(d u)(d v)[(d u)+(d v)],
$$

to generalize the Zagreb indices. Table 2 gives mathematical derivations of these indices from $(\alpha, \beta)$-Zagreb index [40]. The authors computed this index for some nanotubes and graphs in [41, 42]. Table 2 relates some of Zagreb types indices with $(\alpha, \beta)$-Zagreb index.

Definition 9. Let $V^{t}$ be a set of words of size $t$ on the set of alphabets of $V$. The letters of word $u$ of length $t$ are denoted by $u_{1}, u_{2}, \ldots, u_{t}$. The concatenation of two words $u$ and $v$ is denoted by $u v$. The graph $S\left(K_{n}, t\right)$, introduced in [43], has vertex set $V^{t}$. An edge exists in $\{u, v\}$ if and only if there exists $i \in\{1,2, \ldots, t\}$ such that $u_{j}=v_{i}$ if $j<i$ and $u_{i} \leq v_{i}$, and $u_{j}=$ $v_{i}$ and $v_{j}=u_{i}$ if $j>i$. For $n=3$, we receive Tower of Hanoi graphs. Later in [44] these graphs have been called as Sierpinski networks.

Sierpinski networks form an extensively studied class of graphs of fractal nature applicable in topology, mathematics of Tower of Hanoi, computer science, and in other disciplines. A large number of properties like physicochemical properties, thermodynamic properties, chemical activity, biological activity, and so on are determined by the chemical applications of graph theory. In [45], the authors computed molecular topological properties of Sierpinski networks and derived closed formulas for the atom-bond connectivity index, geometric-arithmetic index, and fourth and fifth version of these topological indices for Sierpinski networks. In [46], the authors discussed some matrical aspects of these
TABLE 2: Relations of Indices with $(\alpha, \beta)$-Zagreb index.

\begin{tabular}{lc}
\hline Index & $\begin{array}{c}\text { Derivation from } \\
(\alpha, \beta) \text {-Zagreb index }\end{array}$ \\
\hline Refined Zagreb index, ReZM & $Z_{(2,1)}$ \\
General first Zagreb index, $M^{\alpha}$ & $Z_{(\alpha-1,0)}$ \\
Forgotten index, $\mathrm{F}(\mathrm{G})$ & $Z_{(2,0)}$ \\
\hline
\end{tabular}

networks. Recently, the authors computed various versions of degree-based topological indices of some variants of these networks using direct approach [47]. But connectivity polynomials such as the $M$-polynomial and generalized Zagreb Index have not been computed for this network. Connectivity and number of connected components of this network have been computed recently in [48]. Hamiltonicity of Sierpinski networks has been discussed as well in [48]. Recently in [49], the author used harmonic Sierpinski gasket as a geometric configuration of small antennas. In [50], the authors discussed some energies of the Sierpinski gasket. The authors in [51] computed strong metric dimension of generalized $S_{k}^{n}$ having isolated pendant nodes. Total colorings of $S_{k}^{n}$ were discussed in [52]. The authors in [53] discussed all kinds of shortest paths in Sierpinski networks with the help of newly designed algorithm. For comprehensive survey and classifications of $S_{k}^{n}$, we refer the readers to [54]. In this paper, we compute $M$-polynomial and some degreebased topological indices of Sierpinski graphs $S_{k}^{n}$. The Sierpinski network $S_{3}^{n}$ has $3^{n}, n \geq 2$ vertices and $3\left(3^{n}-1\right) / 2$ edges. Figure 1 shows $S_{3}^{2}$ where $S_{3}$ is triangle.

Now, we present $S_{3}^{4}$ in Figure 2 .

The Sierpinski network $S_{4}^{n}$ has $4^{n}, n \geq 2$ vertices and $4\left(4^{n}-1\right) / 3$ edges. Figure 3 depicts first two instances of Sierpinski network $S_{4}^{n}$ for $n=1$ and $n=2$.

Note that $S_{3}^{n}$ and $S_{4}^{n}$ contain only vertices of degrees 2 and 3. Edge partition of $S(n, k)$ Sierpinski network is based on degrees of end vertices of each edge.

\section{Main Results}

In this section, we give $M$-polynomial, related indices, and some general Zagreb indices of Sierpinski networks $S_{k}^{n}$.

Theorem 1. The M-polynomial of $S_{k}^{n}, n \geq 2$, is $M\left(S_{k}^{n} ; x, y\right)=2 k x^{k-1} y^{k}+\left(\left(k^{n+1}-5 k\right) / 2\right) x^{k} y^{k}$. 


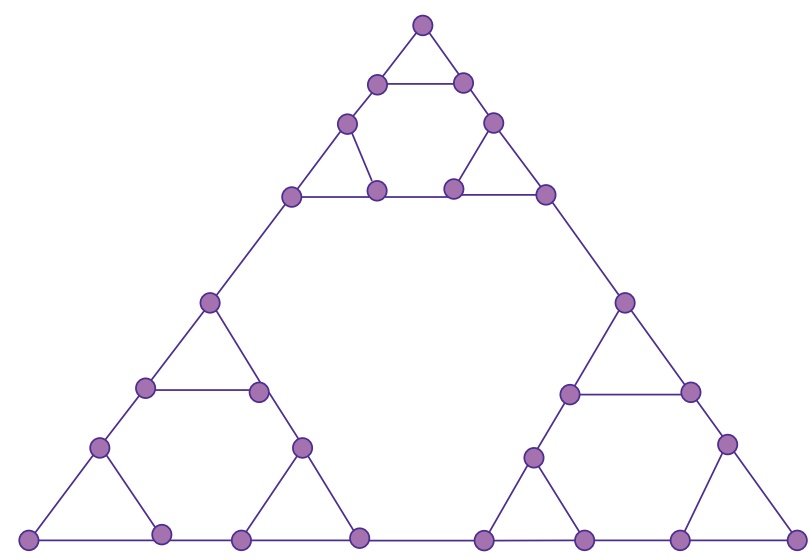

Figure 1: $S_{3}^{2}$.

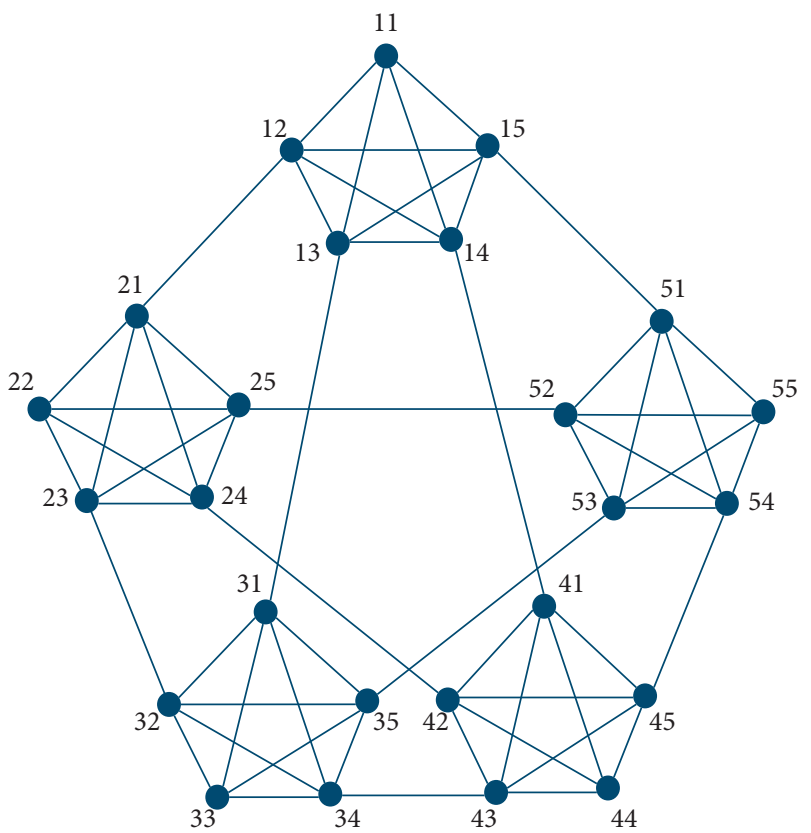

Figure 2: $S_{3}^{4}$.

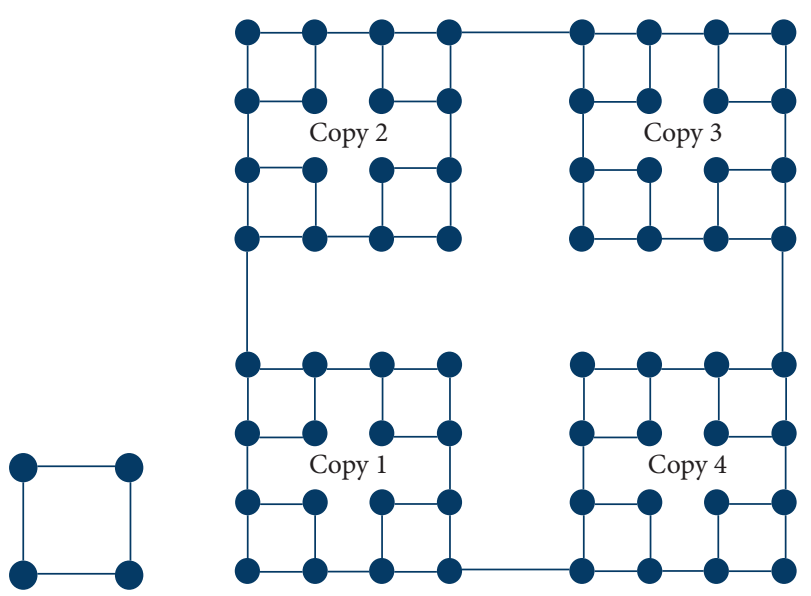

Figure 3: $S_{4}$ and $S_{4}^{2}$.
Proof. We prove it by finding sets that partition vertices and edges of $S_{k}^{n}$. The disjoint sets of vertices of $S_{k}^{n}$ are

$$
\begin{aligned}
V_{\{k-1\}} & =\left\{v \mathcal{E} V(G) \mid d_{v}=2\right\} \longrightarrow\left|V_{\{1\}}\right|=k, \\
V_{\{k\}} & =\left\{v \mathcal{E} V(G) \mid d_{v}=3\right\} \longrightarrow\left|V_{\{2\}}\right|=k^{n}-k .
\end{aligned}
$$

The edge partition of $S_{k}^{n}$ contains the sets

$$
\begin{aligned}
E_{\{k-1, k\}} & =\left\{e=u v \varepsilon E\left(S_{k}^{n}\right) \mid d_{u}=2, d_{v}=k\right\} \longrightarrow\left|E_{\{2, k\}}\right|=2 k, \\
E_{\{k, k\}} & =\left\{e=u v \varepsilon E\left(S_{k}^{n}\right) \mid d_{u}=3, d_{v}=3\right\} \longrightarrow\left|E_{\{k, k\}}\right| \\
& =\frac{k^{n+1}-5 k}{2} .
\end{aligned}
$$

Now the $M$-polynomial of $S_{k}^{n}$ is

$$
\begin{aligned}
M\left(S_{k}^{n} ; x, y\right) & =\sum_{i \leq j} m_{i j} x^{i} y^{j} \\
& =\sum_{2 \leq k} m_{2 k} x^{k-1} y^{k}+\sum_{k \leq k} m_{k k} x^{k} y^{k} \\
& =\sum_{u v \varepsilon E_{\{2, k\}}} m_{2 k} x^{k-1} y^{k}+\sum_{u v \varepsilon E_{\{k, k\}}} m_{k k} x^{k} y^{k} \\
& =\left|E_{\{2, k\}}\right| x^{k-1} y^{k}+\left|E_{\{k, k\}}\right| x^{k} y^{k} \\
& =2 k x^{k-1} y^{k}+\left(\frac{k^{n+1}-5 k}{2}\right) x^{k} y^{k} .
\end{aligned}
$$

Theorem 2. The first Zagreb, second Zagreb, modified second Zagreb, Randic, reciprocal generalized Randić, and symmetric division indices of $S_{k}^{n}, n \geq 2$, are

(1) $M_{1}\left(S_{k}^{n}\right)=4 k^{2}-2 k+k\left(k^{n+1}-5 k\right)$

(2) $M_{2}\left(S_{k}^{n}\right)=2 k^{2}(k-1)+k^{2}\left(\left(k^{n+1}-5 k\right) / 2\right)$

(3) $m M_{2}\left(S_{k}^{n}\right)=(2 /(k-1))+\left(\left(k^{n}-5\right) / 2 k\right)$

(4) $R \alpha\left(S_{k}^{n}\right)=2 k^{\alpha+1}(k-1)^{\alpha}+k^{2 \alpha}\left(\left(k^{n+1}-5 k\right) / 2\right)$ 
(5) $\operatorname{RR} \alpha\left(S_{k}^{n}\right)=\left(2 / k^{\alpha-1}\right)+\left(k^{n+1} / 2 k^{2 \alpha}\right)$

(6) $\operatorname{SDD}\left(S_{k}^{n}\right)=\left(\left(2(k-1)^{2}+2 k^{2}\right) /(k-1)\right)+k^{n+1}-5 k$
Proof. Before giving step-by-step proofs of all items, we find $D_{x}, D_{y}, S_{x}$, and $S_{y}$.

$$
\begin{aligned}
D_{x}\left(S_{k}^{n}\right) & =2 k(k-1) x^{k-1} y^{k}+k\left(\frac{k^{n+1}-5 k}{2}\right) x^{k} y^{k}, \\
D_{y}\left(S_{k}^{n}\right) & =2 k^{2} x^{k-1} y^{k}+k\left(\frac{k^{n+1}-5 k}{2}\right) x^{k} y^{k}, \\
S_{x}\left(S_{k}^{n}\right) & =\frac{2 k}{k-1} x^{k-1} y^{k}+\frac{1}{k}\left(\frac{k^{n+1}-5 k}{2}\right) x^{k} y^{k}, \\
S_{y}\left(S_{k}^{n}\right) & =2 x^{k-1} y^{k}+\frac{1}{k}\left(\frac{k^{n+1}-5 k}{2}\right) x^{k} y^{k}, \\
D_{x} D_{y}\left(M\left(S_{k}^{n}\right)\right) & =2 k^{2}(k-1) x^{k-1} y^{k}+k^{2}\left(\frac{k^{n+1}-5 k}{2}\right) x^{k} y^{k}, \\
\left(D_{x}+D_{y}\right)\left(M\left(S_{k}^{n}\right)\right) & =4 k^{2} x^{k-1} y^{k}+2 k\left(\frac{k^{n+1}-5 k}{2}\right) x^{k} y^{k}, \\
S_{x} S_{y}\left(M\left(S_{k}^{n}\right)\right) & =\frac{2}{k-1} x^{k-1} y^{k}+\left(\frac{k^{n}-5}{2 k}\right) x^{k} y^{k}, \\
D_{x}^{\alpha} D_{y}^{\alpha}\left(M\left(S_{k}^{n}\right)\right) & =2 k^{\alpha+1}(k-1)^{\alpha} x^{k-1} y^{k}+k^{2 \alpha}\left(\frac{k^{n+1}-5 k}{2}\right) x^{k} y^{k}, \\
S_{x}^{\alpha} S_{y}^{\alpha}\left(M\left(S_{k}^{n}\right)\right) & =\frac{2 x^{k-1} y_{y}^{k}}{k^{\alpha-1}+\frac{k^{n+1}}{2 k^{2 \alpha}} x^{k} y^{k},}\left(M\left(S_{k}^{n}\right)\right) \\
& =\left(\frac{2(k-1)^{2}+2 k^{2}}{k-1}\right) x^{k-1} y^{k}+2 k\left(\frac{k^{n+1}-5 k}{2}\right) x^{k} y^{k} .
\end{aligned}
$$

Now the indices are

(1) $M_{1}\left(S_{k}^{n}\right)=\left.\left(D_{x}+D_{y}\right)\left(M\left(S_{k}^{n}\right)\right)\right|_{x=y=1}=k^{n+2}-k^{2}-2 k$

(2) $M_{2}\left(S_{k}^{n}\right)=\left.\left(D_{x} D_{y}\right)\left(M\left(S_{k}^{n}\right)\right)\right|_{x=y=1}=2 k^{2}(k-1)+$ $k^{2}\left(\left(k^{n+1}-5 k\right) / 2\right)$

(3) $m M_{2}\left(S_{k}^{n}\right)=\left.\left(S_{x} S_{y}\right)\left(M\left(S_{k}^{n}\right)\right)\right|_{x=y=1}=(2 /(k-1))+$ $\left(\left(k^{n}-5\right) / 2 k\right)$

(4) $R \alpha\left(S_{k}^{n}\right)=\left.\left(D_{x}^{\alpha} D_{y}^{\alpha}\right)\left(M\left(S_{k}^{n}\right)\right)\right|_{x=y=1}=2 k^{\alpha+1}(k-1)^{\alpha}$ $+k^{2 \alpha}\left(\left(k^{n+1}-5 k\right) / 2\right)$

(5) $R R \alpha\left(S_{k}^{n}\right)=\left.\left(S_{x}^{\alpha} S_{y}^{\alpha}\right)\left(M\left(S_{k}^{n}\right)\right)\right|_{x=y=1}=$ $\left(2 /(k-1)^{\alpha}(k)^{\alpha-1}\right)+\left(\left(k^{n+1}-5 k\right) / 2 k^{2 \alpha}\right)$

(6) $\operatorname{SDD}\left(S_{k}^{n}\right)=\left.\left(D_{x} S_{y}+S_{x} D_{y}\right)\left(M\left(S_{3}^{n}\right)\right)\right|_{x=y=1}=$ $\left(\left(2(k-1)^{2}+2 k^{2}\right) /(k-1)\right)+k^{n+1}-5 k$

Theorem 3. The harmonic, inverse sum, and augmented indices of $S_{k}^{n}, n \geq 1$, are

(1) $H\left(S_{k}^{n}\right)=((4 k) /(2 k-1))+\left(\left(k^{n+1}-5 k\right) /(2 k)\right)$

(2) $I\left(S_{k}^{n}\right)=\left(\left(2 k^{2}(k-1)\right) /(2 k-1)\right)+\left(k\left(k^{n+1}-5 k\right) / 4\right)$
(3) $A\left(S_{k}^{n}\right)=\left(\left(2 k^{4}(k-1)^{3}\right) /\left((2 k-3)^{3}\right)\right)+$ $\left(k^{6} /(2 k-2)^{3}\right)\left(\left(k^{n+1}-5 k\right) / 2\right)$

Proof

(1) $H\left(S_{k}^{n}\right)=2 S_{x} J\left(S_{k}^{n} ; x, y\right)_{\mid x=1}=\left[2 S_{x}\left(2 k x^{2 k-1}+\right.\right.$ $\left.\left.\left(\left(k^{n+1}-5 k\right) / 2\right) x^{2} k\right)\right]_{x=1}=2\left[((2 k) /(k-1)) x^{2 k-1}+\right.$ $\left.(1 / 2 k)\left(\left(k^{n+1}-5 k\right) / 2\right) x^{2} k\right]_{x=1}=((4 k) /(2 k-1))+$ $\left(\left(k^{n+1}-5 k\right) /(2 k)\right)$

(2) $I\left(S_{k}^{n}\right)=\left[S_{x} J D_{x} D_{y}\left(S_{k}^{n}\right)\right]_{x=1}=\left[S_{x} J D_{x}\left(D_{y}\left(S_{k}^{n}\right)\right)\right]_{x=1}$ $=\left[S_{x} J D_{x} 2 k^{2} x^{k-1} y^{k}+k\left(\left(k^{n+1}-5 k\right) / 2\right) x^{k} y^{k}\right]_{x=1}=$ $\left[S_{x} J 2 k^{2}(k-1) x^{k-1} y^{k}+k^{2}\left(\left(k^{n+1}-5 k\right) / 2\right) x^{k} y^{k}\right]_{x=1}=$ $\left.\left[S_{x} 2 k^{2}(k-1)\right) x^{2} k-1+k^{2}\left(\left(k^{n+1}-5 k\right) / 2 x^{2 k}\right)\right]_{x=1}=$ $\left[\left(\left(2 k^{2}(k-1) 2 k-1\right) /\left(x^{2 k-1}\right)\right)+k\left(\left(k^{n+1}-5 k\right) /\right.\right.$ 4) $\left.x^{2 k}\right]_{x=1}=\left(\left(2 k^{2}(k-1)\right) /(2 k-1)\right)+k\left(\left(k^{n+1}-5 k\right) / 4\right)$

(3) $A\left(S_{k}^{n}\right)=\left[S_{x}^{3} Q_{-2} J D_{x}^{3} D_{y}^{3}\left(M\left(S_{k}^{n}\right)\right)\right]_{x=1}=$ $\left[S_{x}^{3} Q_{-2} J\left(2 k^{4}(k-1)^{3}\right) x^{2 k-1}+k^{6} x^{2 k}\left(k^{n+1}-5 k / 2\right)\right]_{x=1}=$ $\left[S_{x}^{3} Q_{-2}\left(2 k^{4}(k-1)^{3}\right) x^{2 k-3}+k^{6} x^{2 k-2}\left(k^{n+1}-5 k / 2\right)\right]_{x=1}$ 


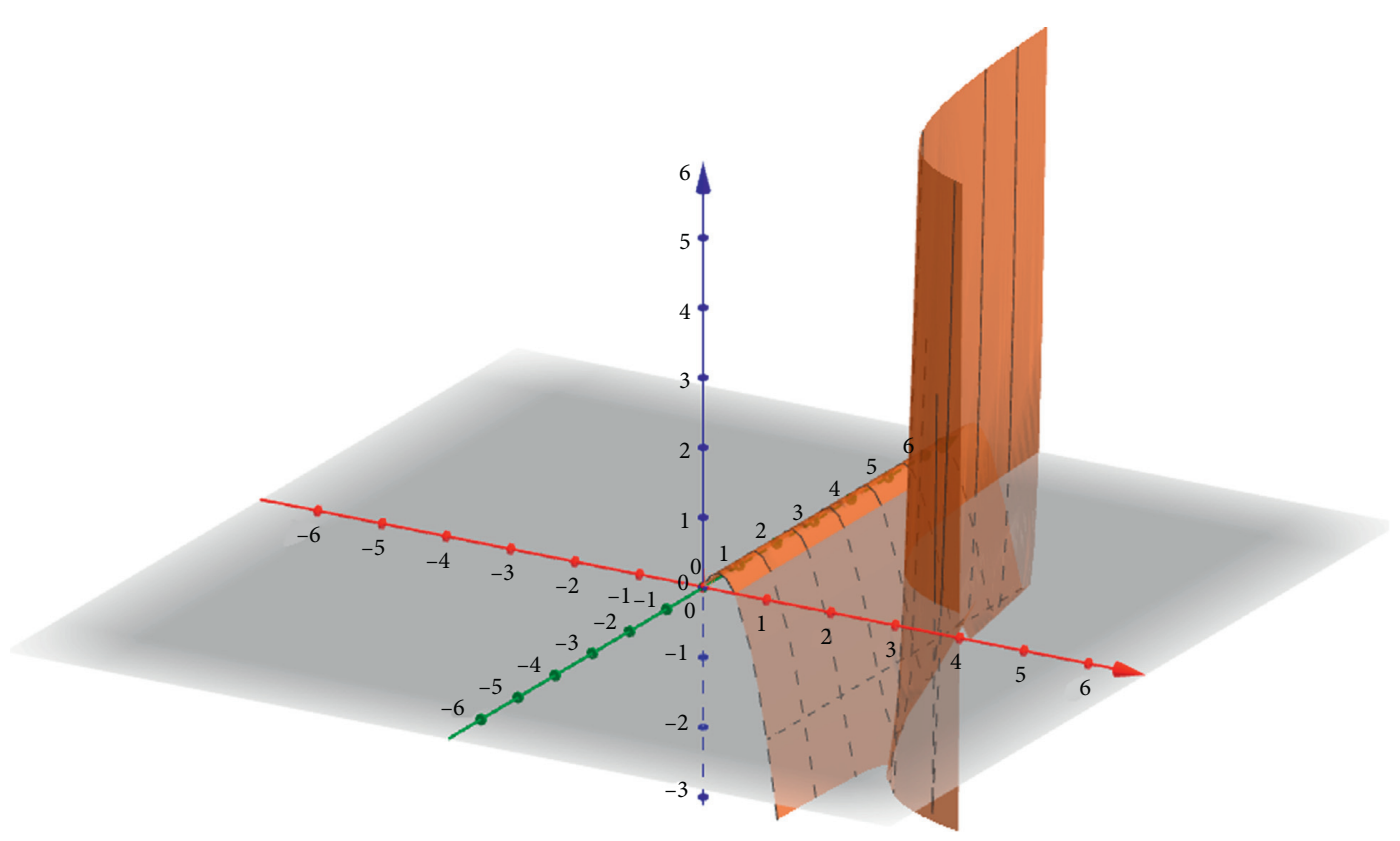

FIGURE 4: $F\left(S_{n}^{k}\right)$.

$$
\begin{aligned}
& =\left[S_{x}^{3}\left(\left(2 k^{4}(k-1)^{3}\right) x^{2 k-3} /(2 k-3)^{3}\right)+k^{6}\left(\left(x^{2 k-2}\right) /\right.\right. \\
& \left.\left.(2 k-2)^{3}\right)\left(k^{n+1}-5 k / 2\right)\right]_{x=1}=\left[\left(\left(2 k^{4}(k-1)^{3}\right) /\right.\right. \\
& \left.(2 k-3)^{3}\right) x^{2 k-3}+k^{6}\left(\left(x^{2 k-2}\right) /(2 k-2)^{3}\right) \\
& \left.\left(k^{n+1}-5 k / 2\right)\right]_{x=1}=\left[\left(\left(2 k^{4}(k-1)^{3}\right) /(2 k-3)^{3}\right)+\right. \\
& \left.k^{6}\left(1 /(2 k-2)^{3}\right)\left(\left(k^{n+1}-5 k\right) / 2\right)\right]
\end{aligned}
$$

Theorem 4. The first Zagreb polynomial, second Zagreb polynomial, forgotten polynomial, and forgotten index of $S_{3}^{n}, n \geq 2$, are

(1) $M_{1}\left(S_{k}^{n} ; x\right)=4 k^{2} x^{2 k-1}+2 k\left(\left(k^{n+1}-5 k\right) / 2\right) x^{2} k$

(2) $M_{2}\left(S_{k}^{n} ; x\right)=2 k^{2}(k-1) x^{2 k-1}+k^{2}\left(\left(k^{n+1}-5 k\right) / 2\right) x^{2 k}$

(3) $F\left(S_{k}^{n} ; x\right)=2 k x^{(k-1)^{2}+(k)^{2}}+\left(\left(k^{n+1}-5 k\right) / 2\right) x^{2 k^{2}}$

(4) $F\left(S_{k}^{n}\right)=2 k\left((k-1)^{2}+(k)^{2}\right)+2 k^{2}\left(\left(k^{n+1}-5 k\right) / 2\right)$

Proof

(1) $M_{1}\left(S_{k}^{n} ; x\right)=\sum_{u v \varepsilon E\left(S_{k}^{n}\right)} x^{\left[d_{u}+d_{v}\right]}=\sum_{u v \varepsilon E_{1}\left(S_{k}^{n}\right)} x^{\left[d_{u}+d_{v}\right]}+$ $\sum_{u v \varepsilon E_{2}\left(S_{k}^{n}\right)} x^{\left[d_{u}+d_{v}\right]}=\left|E_{1}\left(S_{k}^{n}\right)\right| x^{2 k-1}+\left|E_{2}\left(S_{k}^{n}\right)\right| x^{2 k}=$ $2 k x^{2 k-1}+\left(\left(k^{n+1}-5 k\right) / 2\right) x^{2 k}$

(2) $M_{2}\left(S_{k}^{n} ; x\right)=\sum_{u v \varepsilon E\left(S_{k}^{n}\right)} x^{\left[d_{u} \times d_{v}\right]}=\sum_{u v \varepsilon E_{1}\left(S_{k}^{n}\right)} x^{\left[d_{u} \times d_{v}\right]}+$ $\sum_{u v \mathcal{E} E_{2}\left(S_{k}^{n}\right)} x^{\left[d_{u} \times d_{v}\right]}=\left|E_{1}\left(S_{k}^{n}\right)\right| x^{2 k-1}+\left|E_{2}\left(S_{k}^{n}\right)\right| x^{2 k}=$ $2 k^{2}(k-1) x^{2 k-1}+k^{2}\left(\left(k^{n+1}-5 k\right) / 2\right) x^{2 k}$

(3) $F\left(S_{k}^{n} ; x\right)=\sum_{u v \varepsilon E\left(S_{k}^{n}\right)} x^{\left[d_{u}^{2}+d_{v}^{2}\right]}=\sum_{u v \varepsilon E_{1}\left(S_{k}^{n}\right)} x^{\left[d_{u}^{2}+d_{v}^{2}\right]}+$ $\sum_{u v \varepsilon E_{2}\left(S_{k}^{n}\right)} x^{\left[d_{u}^{2}+d_{v}^{2}\right]}=\left|E_{1}\left(S_{k}^{n}\right)\right| x^{(k-1)^{2}+(k)^{k^{2}}}+$ $\left|E_{2}\left(S_{k}^{n}\right)\right| x^{2 k^{2}}=2 k x^{(k-1)^{2}+(k)^{2}}+\left(\left(k^{n+1}-5 k\right) / 2\right) x^{2 k^{2}}$

(4) $F\left(S_{k}^{n}\right)=\sum_{u v \varepsilon E\left(S_{k}^{n}\right)}\left[d_{u}^{2}+d_{v}^{2}\right]=\sum_{u v \varepsilon E_{1}\left(S_{k}^{n}\right)}\left[d_{u}^{2}+d_{v}^{2}\right]+$ $\sum_{u v \varepsilon E_{2}\left(S_{k}^{n}\right)}\left[d_{u}^{2}+d_{v}^{2}\right]=(k-1)^{2}+(k)^{2}\left|E_{1}\left(S_{k}^{n}\right)\right|+$
$2 k^{2}\left|E_{2}\left(S_{k}^{n}\right)\right|=2 k\left((k-1)^{2}+(k)^{2}\right)+$ $2 k^{2}\left(\left(k^{n+1}-5 k\right) / 2\right)$

Theorem 5. For all $S_{k}^{n}, n \geq 1$, we have $Z_{\alpha, \beta}=2(k-1)^{\alpha} k^{\beta+1}+\left(\left(k^{n}-5\right) / 2\right) k^{\alpha+\beta+1}$.

$$
\begin{gathered}
\operatorname{REZ} \alpha\left(S_{k}^{n}\right)=Z_{2,1}=k^{2}\left[2(k-1)^{2}+\left(\frac{k^{n}-5}{2}\right) k^{2}\right], \\
M^{\alpha}\left(S_{k}^{n}\right)=Z_{\alpha-1,0}=\left[2 k(k-1)^{\alpha-1}+\left(\frac{k^{n}-5}{2}\right) k^{\alpha}\right], \\
F\left(S_{k}^{n}\right)=Z_{2,0}=k\left[2(k-1)^{2}+\left(\frac{k^{n}-5}{2}\right) k^{2}\right] .
\end{gathered}
$$

Proof. To prove this, we again have to refer to Table 1 where edge partitions of $S_{k}^{n}, n \geq 1$ are given. Then, by using relation in (2), we have

$$
\begin{aligned}
Z_{\alpha, \beta} & =\sum_{u v \in E(G)}\left[(d u)^{\alpha}(d v)^{\beta}+(d u)^{\beta}(d v)^{\alpha}\right] \\
& =2 k(k-1)^{\alpha}(k)^{\beta}+\left(\frac{k^{n+1}-5 k}{2}\right) k^{\alpha} k^{\beta} \\
& =2 k(k-1)^{\alpha}(k)^{\beta}+\left(\frac{k^{n}-5}{2}\right) k^{\alpha+\beta+1} .
\end{aligned}
$$

Remaining three parts of this theorem are just easy consequences of the result of $Z_{\alpha, \beta}$. 


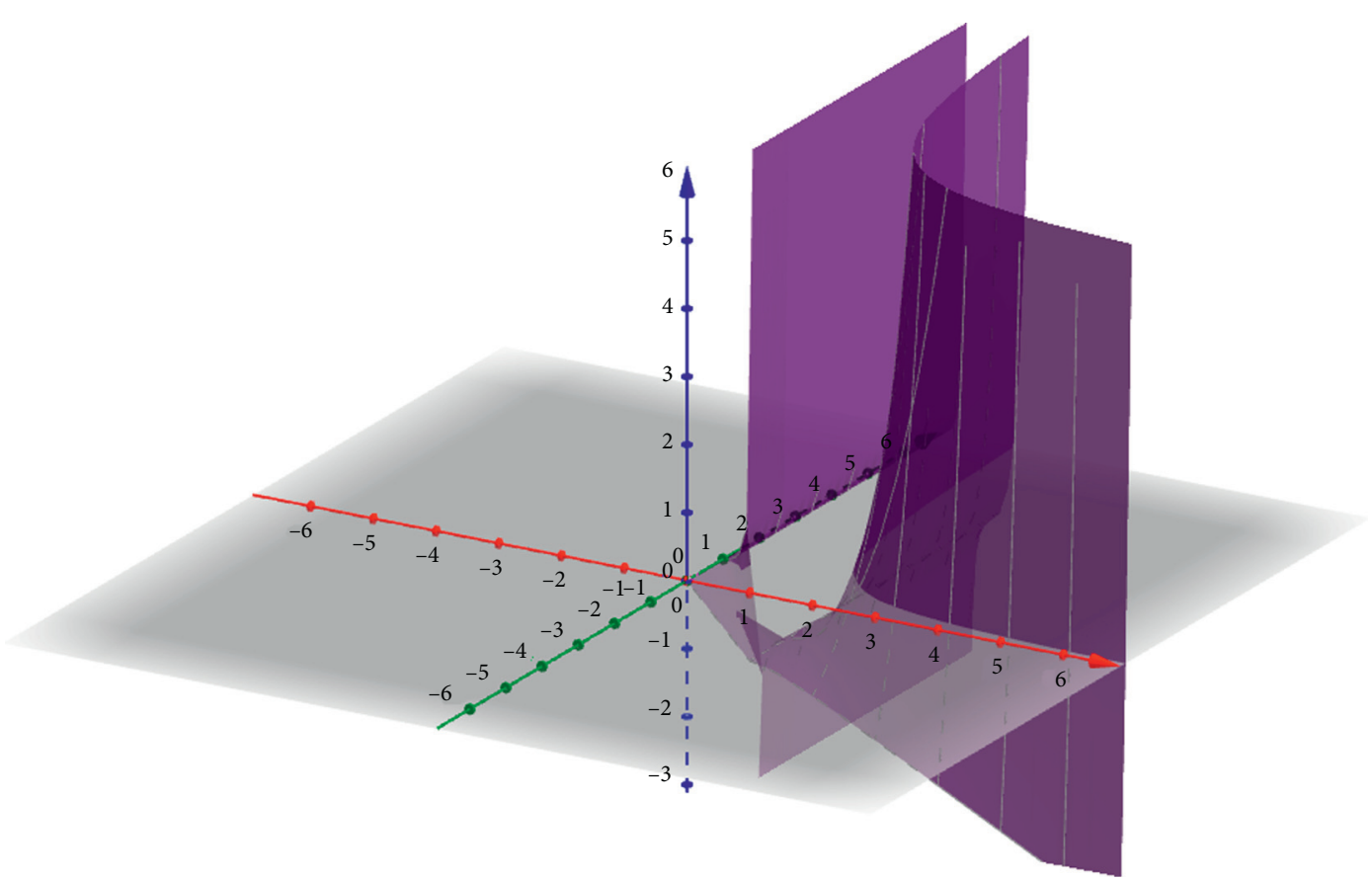

Figure 5: Inverse sum index of $\left(S_{n}^{k}\right)$.

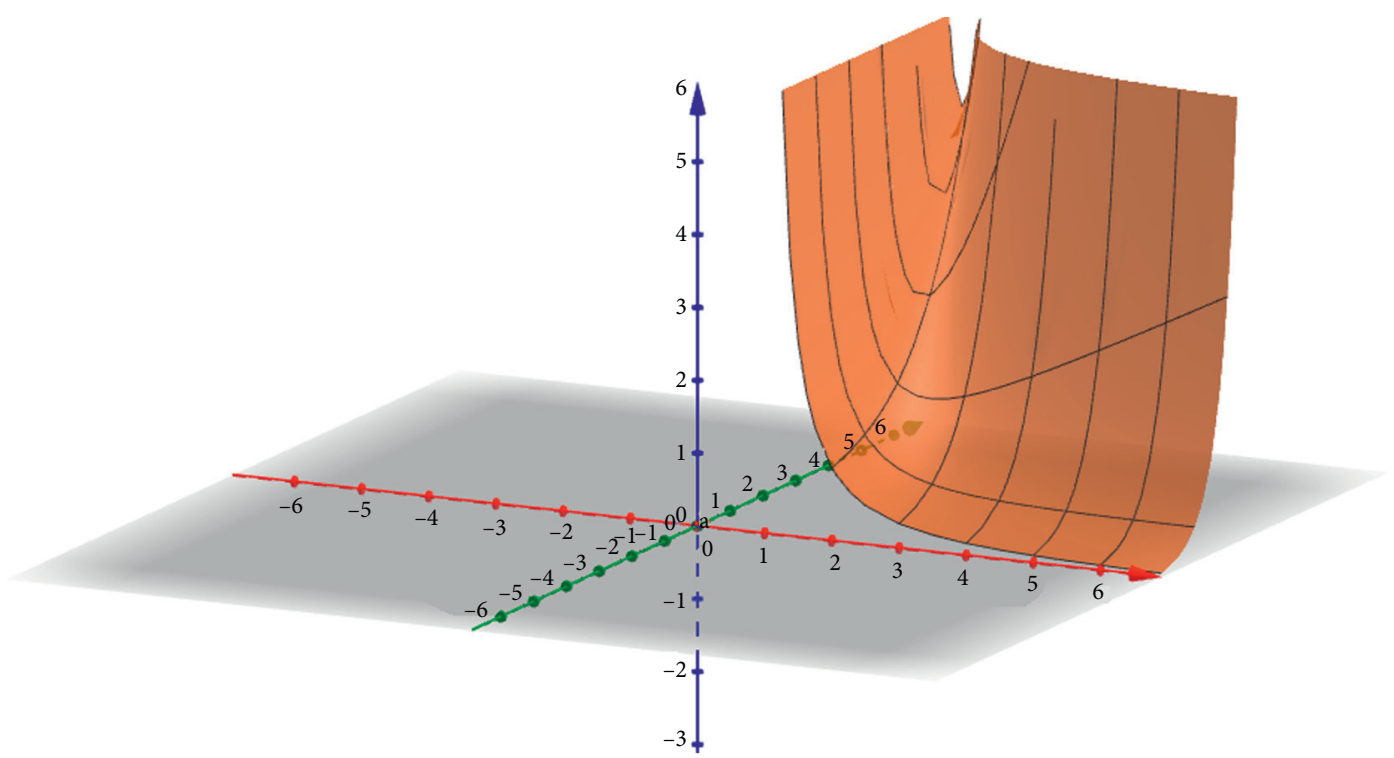

Figure 6: $m M_{2}\left(S_{n}^{k}\right)$.

\section{Conclusions and Discussion}

In the present article, we computed some connectivity polynomials and many degree-based descriptors of Sierpinski networks specifically the closed form of $M$-polynomial and generalized Zagreb index. In this section, we give graphical representation of our results. Figures 4-7 are graphic index analysis of Sierpinski networks. These graphs are self explanatory to some extent and clearly demonstrate that values of all indices depend directly on the parameters $n$ and $k$. We pose natural open problem to compute all above indices and polynomials for more generalized kinds of Sierpinski networks $S(G, K)$ where $G$ is any graph. We here give short analysis of the indices depending upon $n$ and $k$. For analysis, we use 3D graphs developed by GeoGebra. First 3D surface is plotted for forgotten index (see Figure 4). Slices along different axes represent the forgotten index for different values of $n$ and $k$.

Next Figure 5 is plotted for inverse sum index. It can be seen that the surface has some part as of a saddle where along one parameter its curvature is opposite to the other parameter.

Figure 6 is the plotted surface for modified second Zagreb index of $\left(S_{n}^{k}\right)$. Here the surface is parabolic cylinder and curves on the surface show the curve for modified 


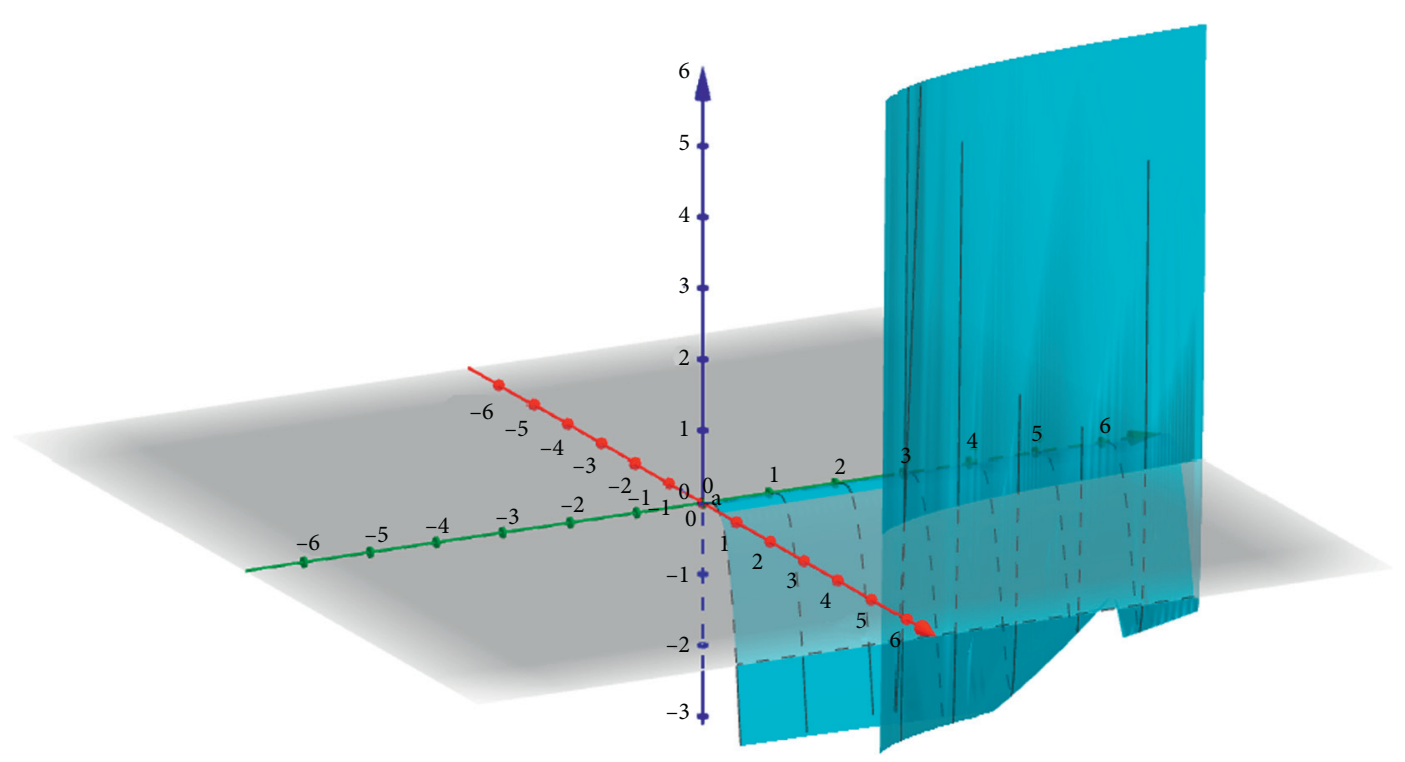

Figure 7: $\operatorname{ReZM}\left(S_{n}^{k}\right)$.

second Zagreb index for different combinations of parameters $n$ and $k$. These curves are parabolic in nature which depict the behaviour of the respective index with respect to particular parameters involved.

Similarly, Figure 7 represents the surface plotted for redefined Zagreb index of $\left(S_{n}^{k}\right)$. Different graphs shown on the surface represent critical behaviour of this index for the parameters $n$ and $k$. It is trivially observed that for some combinations, it is a straight line, and for some, it is parabolic. These facts can be used as raw inputs in networking and fractals where these networks play a significant role. All surfaces for other computed indices can be easily plotted and interpreted similarly. Physicochemical properties of the molecules modelled on the $S_{n}^{k}$ networks can be forecasted using the results given above.

\section{Data Availability}

No data were used to support this study.

\section{Conflicts of Interest}

The authors declare that they have no conflicts of interest.

\section{Authors' Contributions}

The manuscript was drafted and written by MMM and $\mathrm{ZH}$. The concepts were initially developed by JBL, CF, and MA. All authors have read and approved the final manuscript.

\section{Acknowledgments}

This study was supported by the Key Teaching Research Project of Quality Engineering in Colleges and Universities in Anhui Province (Research on Teaching Management Reform and Innovation in Higher Vocational Colleges Based on Big Data) (subject no. 2017jyxm0907).

\section{References}

[1] Y. Qi and Z. Zhang, "Spectral properties of extended sierpiński graphs and their applications," IEEE Transactions on Network Science and Engineering, vol. 6, no. 3, p. 512, 2019.

[2] E. Deutsch and S. Kavžar, " $M$-polynomial and degree-based topological indices. Iran," Journal of Mathematical Chemistry, vol. 6, pp. 93-102, 2015.

[3] K. C. Das, K. Xu, and J. Nam, "Zagreb indices of graphs," Frontiers of Mathematics in China, vol. 10, no. 3, pp. 567-582, 2015.

[4] I. Gutman, "Degree-based topological indices," Croatica Chemica Acta, vol. 86, no. 4, pp. 351-361, 2013.

[5] I. Gutman and J. Tosovic, "Testing the quality of molecular structure descriptors. Vertex-degree-based topological indices," Journal of the Serbian Chemical Society, vol. 78, no. 6, pp. 805-810, 2013.

[6] M. H. Khalifeh, H. Yousefi-Azari, and A. R. Ashrafi, "The first and second zagreb indices of some graph operations," Discrete Applied Mathematics, vol. 157, no. 4, pp. 804-811, 2009.

[7] K. Xu, K. Tang, H. Liu, and J. Wang, "The zagreb indices of bipartite graphs with more edges," Journal of Applied Mathematics \& Informatics, vol. 33, no. 3_4, pp. 365-377, 2015.

[8] B. Zhou and I. Gutman, "Further properties of zagreb indices," MATCH Communications in Mathematical and in Computer Chemistry, vol. 54, pp. 233-239, 2005.

[9] B. Zhou, "Upper bounds for the zagreb indices and the spectral radius of series-parallel graphs," International Journal of Quantum Chemistry, vol. 107, no. 4, pp. 875-878, 2007.

[10] M. Munir, W. Nazeer, A. R. Nizami, S. Rafique, and S. M. Kang, "M-polynomials and topological indices of titania nanotubes," Syemmetry, vol. 8, pp. 1-9, 2017.

[11] M. Munir, W. Nazeer, S. Rafique, A. R. Nizami, and S. M. Kang, "Some computational aspects of triangular Boron nanotubes," Symmetry, vol. 9, pp. 1-11, 2017.

[12] M. Munir, W. Nazeer, S. M. Kang, M. I. Qureshi, A. R. Nizami, and Y. C. Kwun, "Some invariants of jahangir graphs," Symmetry, vol. 9, pp. 1-15, 2017. 
[13] M. Ajmal, W. Nazeer, M. Munir, S. M. Kang, and C. Y. Jung, "The $M$-polynomials and topological indices of generalized prism network," International Journal of Mathematical Analysis, vol. 11, pp. 293-303, 2017.

[14] A. Ashaq, W. Nazeer, M. Munir, and S. M. Kang, " $M-$ polynomials and topological indices of zigzag and rhombic benzenoid systems," Open Chemistry, vol. 16, pp. 73-78, 2018.

[15] S. M. Kang, W. Nazeer, M. A. Zahid, A. R. Nizami, A. Aslam, and M. Munir, "M-polynomials and topological indices of hex-derived networks," Open Physics, vol. 16, no. 1, pp. 394-403, 2018.

[16] Y. C. Kwun, M. Munir, W. Nazeer, S. Rafique, and S. M. Kang, "M-Polynomials and topological indices of V-Phenylenic Nanotubes and Nanotori," Nature Scitific Reports, vol. 7, 2017.

[17] M. Randic, "Characterization of molecular branching," Journal of the American Chemical Society, vol. 97, no. 23, pp. 6609-6615, 1975.

[18] I. Gutman and B. Furtula, Recent Results in the Theory of Randic Index, MCM Worldwide, Munich, Germany, 2008.

[19] X. Li and I. Gutman, Mathematical Aspects of Randic-type Descriptors, MCM Worldwide, Munich, Germany, 2006.

[20] R. Todeschini and V. Consonni, Handbook of Molecular Descriptors, Wiley-VCH, Weinheim, Germany, 2000.

[21] D. Amic, D. Beslo, B. Lucic, S. Nikolic, and N. Trinajstić, "The vertex-connectivity index revisited," Journal of Chemical Information and Modeling, vol. 38, pp. 819-822, 1998.

[22] B. Bollobas and P. Erdos, "Graphs of extremal weights," Ars Combinatoria, vol. 50, pp. 225-233, 1998.

[23] X. Li and T. Gutman, Mathematical Chemistry Monographs, MCM Worldwide, Munich, Germany, 2006.

[24] Y. Huang, X. Li, Y. Shi, T. Xu, and I. Gutman, "On molecular graphs with smallest and greatest zeroth-Corder general randic index," MATCH Communications in Mathematical and in Computer Chemistry, vol. 54, pp. 425-434, 2005.

[25] I. Gutman and N. Trinajstić, "Graph theory and molecular orbitals. Total $\varphi$-electron energy of alternant hydrocarbons," Chemical Physics Letters, vol. 17, no. 4, pp. 535-538, 1972.

[26] B. Furtula, A. Graovac, and D. Vukičević, "Augmented zagreb index," Journal of Mathematical Chemistry, vol. 48, no. 2, pp. 370-380, 2010.

[27] Y. Huang, B. Liu, and L. Gan, "Augmented zagreb index of connected graphs," MATCH Communications in Mathematical and in Computer Chemistry, vol. 67, pp. 483-494, 2012.

[28] J. Braun, A. Kerber, M. Meringer, and C. Rucker, "Similarity of molecular descriptors the equivalence of zagreb indices and walk counts," MATCH Communications in Mathematical and in Computer Chemistry, vol. 54, pp. 163-176, 2005.

[29] S. Fajtlowicz, "On conjectures of graffiti-II," Congr. Numer, vol. 60, pp. 187-197, 1987.

[30] I. Gutman and K. C. Das, "The first zagreb index 30 Years after," MATCH Communications in Mathematical and in Computer Chemistry, vol. 50, pp. 83-92, 2004.

[31] S. Nikolic, G. Kovacevic, A. Milicevic, and N. Trinajstic, "The zagreb indices 30 Years after," Croatica Chemica Acta, vol. 76, pp. 113-124, 2003.

[32] X. Li and Y. Shi, "A survey on the Randic index," MATCH Communications in Mathematical and in Computer Chemistry, vol. 59, pp. 127-156, 2008.
[33] B. Zhou, "Remarks on zagreb indices," MATCH Communications in Mathematical and in Computer Chemistry, vol. 57, pp. 591-596, 2007.

[34] I. Gutman and O. E. Polansky, Mathematical Concepts in Organic Chemistry, Springer, Berlin, Germany, 1986.

[35] M. Azari and A. Iranmanesh, "Generalized Zagreb index of graphs," Studia Universitatis Babes-Bolyai Mathematica, vol. 56, no. 3, pp. 59-70, 2011.

[36] P. Sarkar, N. De, I. N. Cangul, and A. Pal, “The (a,b)-Zagreb index of some derived networks," Journal of Taibah University for Science, vol. 13, no. 1, p. 79, 2019.

[37] M. R. Farahani and M. R. R. Kanna, "Generalized Zagreb index of V-phenylenic nanotubes and nanotori," Journal of Chemical and Pharmaceutical Research, vol. 7, no. 11, pp. 241-245, 2015.

[38] M. R. Farahani, "The generalized Zagreb index of circumcoronene series of benzenoid," Journal of Applied Physical Science International, vol. 3, no. 3, pp. 99-105, 2015.

[39] P. S. Ranjini, V. Lokesha, and A. Usha, "Relation between phenylene and hexagonal squeeze using harmonic index," International Journal of Graph Theory and Its Applications, vol. 1, pp. 116-121, 2013.

[40] B. Basavanagoud, V. R. Desai, and S. Patil, " $(\beta, \alpha)-$ Connectivity index of graphs," Applied Mathematics and Nonlinear Sciences, vol. 2, no. 1, pp. 21-30, 2017.

[41] B. Zhao, J. Gan, and H. Wu, "Redefined Zagreb indices of some nano structures," Applied Mathematics and Nonlinear Sciences, vol. 1, no. 1, pp. 291-300, 2016.

[42] R. P. Kumar, D. S. Nandappa, and M. R. Rajesh Kanna, "Redefined Zagreb, Randic, harmonic and GA indices of graphene," International Journal of Mathematical Analysis, vol. 11, no. 10, pp. 493-502, 2017.

[43] S. Klavzar and U. Milutinovic, "Graphs $S(n, k)$ and a variant of the tower Hanoi problem," Czechoslovak Mathematical Journal, vol. 47, pp. 95-104, 1997.

[44] S. Klavzar, U. Milutionovic, and C. Petr, "1-Perfect codes in sierpinski graphs, bull. Austral,” Mathematics Society, vol. 66, pp. 369-384, 2002.

[45] M. Imran, W. Gao, and M. Reza Farahani, "On topological properties of sierpinski networks," Chaos, Solitons \& Fractals, vol. 98, pp. 199-204, 2017.

[46] C. Luo and L. Zuo, "Metric properties of Sierpin'ski-like graphs," Applied Mathematics and Computation, vol. 296, pp. 124-136, 2017.

[47] M. A. Iqbal, M. Imran, M. K. Siddiqui, and M. A. Zaighum, Computing Molecular Topological Descriptors of Polymeric Networks Modeled by Sierpinski Networks, pp. 1-20, Polycyclic Aromatic Compounds, Taylor and Francis, Milton, UK, 2020.

[48] S. Klavzar and S. Zemljic, "Connectivity and some other properties of generalized Sierpiński graphs," Applicable Analysis and Discrete Mathematics, vol. 12, no. 2, pp. 401-412, 2018.

[49] E. Guariglia, "Harmonic sierpinski gasket and applications," Entropy, vol. 20, no. 9, p. 714, 2018.

[50] J. Azzam, M. A. Hall, and R. S. Strichartz, "Conformal energy, conformal Laplacian, and energy measures on the Sierpinski gasket," Transactions of the American Mathematical Society, vol. 360, pp. 2089-2130, 2008.

[51] E. Estaji and J. A. Rodreguez-Velazquez, "The strong metric dimension of general- ized Sierpinski graphs with pendant vertices," Ars Mathematica Contemporanea, vol. 12, no. 1, pp. 127-134, 2017. 
[52] J. Geetha and K. Somasundaram, "Total coloring of generalized Sierpinski graphs," The Australasian Journal of Combinatorics, vol. 63, pp. 58-69, 2015.

[53] A. M. Hinz and C. Holz auf der Heide, "An efficient algorithm to determine all shortest paths in Sierpiński graphs," Discrete Applied Mathematics, vol. 177, pp. 111-120, 2014.

[54] A. M. Hinz, S. Klavžar, and S. S. Zemljič, "A survey and classification of Sierpiński-type graphs," Discrete Applied Mathematics, vol. 217, no. 3, pp. 565-600, 2017. 